\title{
Nonlinear properties of silicon optical fibers from telecoms to the mid-infrared
}

\author{
A. C. Peacock ${ }^{1}$, P. Mehta ${ }^{1}$, L.Shen ${ }^{1}$, T. D. Day ${ }^{2}$, J. R. Sparks ${ }^{2}$, J. V. Badding ${ }^{2}$, and \\ N. Healy ${ }^{1}$ \\ ${ }^{1}$ Optoelectronics Research Centre, University of Southampton, Southampton \\ SO17 1BJ, UK, ${ }^{2}$ Department of Chemistry and Materials Research Institute, \\ Pennsylvania State University 16802 PA, USA
}

Silicon waveguides are becoming an increasingly popular platform for nonlinear optical signal processing. The incorporation of silicon into the optical fiber geometry provides an important step towards integrating semiconductor functionality with conventional fiber infrastructures, as well as allowing for the construction of robust devices with novel waveguiding properties. In this paper we review our progress in characterizing the nonlinear transmission properties of the hydrogenated amorphous silicon (a-Si:H) core fibers fabricated using a high pressure chemical deposition technique [1]. a-Si:H is emerging as a important material for nonlinear photonics owing to its high nonlinearity, low fabrication costs, and low transmission losses. Although, to date, this material has largely been characterized at telecommunications wavelengths [2], we have recently extended our investigations to present the first characterization of both the nonlinear absorption and refractive index over a wavelength region that crosses the two-photon absorption (TPA) edge, stretching towards the mid-infrared regime. These results will be discussed in relation to future device development, where we will demonstrate the potential for all-optical control using both cross-phase and cross-absorption modulation.
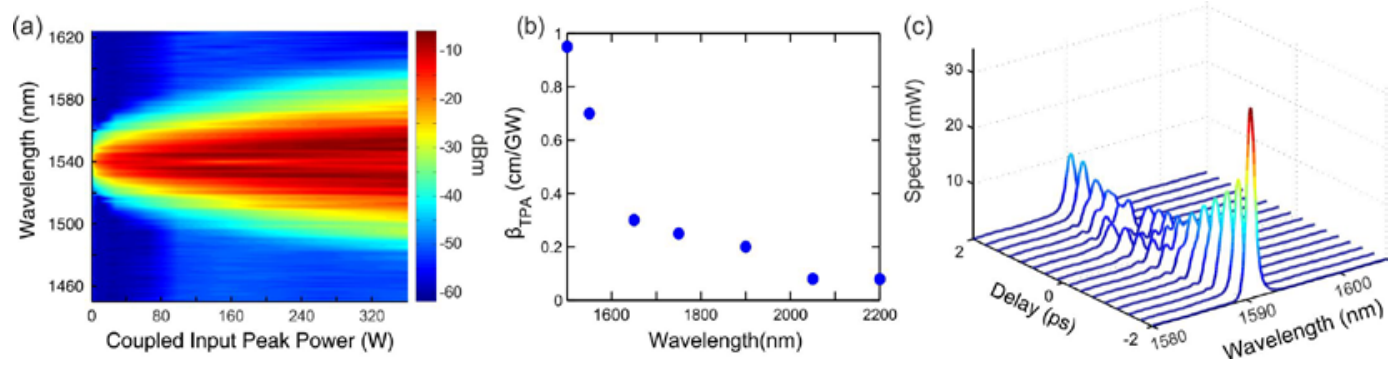

Nonlinear characterization of a a-Si:H core optical fiber: (a) nonlinear spectral broadening, (b) measured TPA parameter, and (c) cross-phase modulation.

[1] P. Mehta, N. Healy, N.F. Baril, P.J.A. Sazio, J.V. Badding, and A.C. Peacock, "Nonlinear transmission properties of hydrogenated amorphous silicon core optical fibers” Opt. Express 16, 16826 (2010).

[2] C. Grillet, L. Carletti, C. Monat, P. Grosse, B. Ben Bakir, S. Menezo, J.M. Fedeli, and D.J. Moss, “Amorphous silicon nanowires combining high nonlinearity, FOM and optical stability,” Opt. Express 20, 22609 (2012). 